\title{
A Formalization of Structured Temporal Objects and Repetition
}

\author{
Diana Cukierman and James Delgrande ${ }^{1}$
}

\begin{abstract}
We propose an approach to formally representing structured temporal objects. These new temporal objects are recursively made up of convex intervals and Allen's convex relations. The major emphasis in our approach is on temporal repetition. To that effect we define the time loop. Time loops allow us to abstract common elements in repetitive temporal patterns. A loop is essentially parameterized by a cycle which is a structured temporal element possibly including subloops, a relation between instances of the cycle and a repetitions number. Atemporal assertions are true during these temporal objects, and thus this formalism allows one to concisely represent and reason with assertions which have an inherent temporal structure. Hence, this formalism allows one to concisely represent, for example, the scheduling of regular meetings in a university, where a meeting may in turn be composed of structured temporal elements.
\end{abstract}

\section{Introduction}

We propose an approach based on convex interval relations [1] to formally representing structured temporal objects. The simplest structured temporal object is a related pair, consisting of two temporal objects with some (interval) relation between them. As well, we have sets of related pairs and time loops. Our primary object of interest is the time loop, used to represent temporal repetition. Atemporal assertions are true during these temporal objects.

The key contribution of this formalism is that we propose a language that treats related intervals (and recursively, related temporal objects) as a structured object, as opposed to a relation among such intervals. This corresponds to the intuition that such structured temporal objects are composite entities, similar for example to physical objects which are made up of different components. In particular, calendar time units are composite temporal objects with a particular structure. Similarly, we view a set of composite and related activities and events (possibly repetitive), for example medical treatments, as occurring during temporally structured objects, and thus "inheriting" such structure.

We abstract (possibly infinite) lists of temporal terms which follow certain repetitive structure with a new temporal term, a loop. We assume that (at some level of abstraction) every occurrence of a repetition or repeat in a sequence of events is "the same"; see [15] for a philosophical analysis. Here we only deal with qualitative information describing a loop repetitive structure. Four parameters provide the necessary information to define a loop: a single (representative) cycle, an order relation between consecutive instances of the cycle or repeats and a repetitions number; the fourth parameter, an index,

\footnotetext{
1 Simon Fraser University, Burnaby, B.C., V5A1S6, Canada, email:\{diana,jim\}@ @s.sfu.ca
}

distinguishes individual loops which have the same structure parameters. A loop cycle can be an interval, but it can also be recursively made up of other (related) intervals and/or other loops.

Previous work in the Artificial Intelligence and Temporal Databases communities have dealt with some sort of structured temporal objects, both interval or point based such as non-convex intervals and linear repeating points; these temporal objects however only provide a "single level" structure. Non-convex intervals are sets of non-intersecting intervals, which "consist intuitively of some (maximal) convex subintervals with convex gaps in between them" [11], page 360. Ligozat's generalized intervals [13] are ordered sequences of points which can also denote non-convex intervals. Linear repeating points, (lrps) are defined within generalized databases. They are mathematical characterization of periodic, and possibly infinite point sequences, with a finite representation [9]. Nearly periodic points [18] and linear repeating intervals (lris) [16] extend lrps. Lris are akin to non-convex intervals where subintervals have the same duration and are equally distanced. Temporal granularities research also deals with structure in time, although focused on calendars and calendar-based time units $[4,6,12,16]$. In contrast, we propose temporal objects which are not calendar based, although they permit one to define calendar-based temporal objects. The language presented here can include any structure with a finite number of nested structured temporal objects, with any qualitative structure; hence a temporal object may include arbitrary gaps. Space limitations preclude that we include more related literature.

The next section introduces the language and presents the proposed axioms for each temporal object, along with examples and some results which follow from the axiomatization. The theory is augmented in Section 3 with the HOLDS predicate and general formulas, with which atemporal assertions are expressed to be true during a certain temporal term. Results are then presented which illustrate the expressive and reasoning power of this theory. Finally, a comparison of this formalism with other work, and comments on current and future research, are provided.

\section{The terms in the language}

The following examples intuitively illustrate the expressive power of temporal terms. Consider two intervals, $s$ and $p$; assume that a sandwich is eaten during $s$ and that a medication pill is taken during $p$. $\langle s, . b ., p\rangle$ stands for a related pair, composed by an interval (during which a sandwich is eaten) before an interval (during which a pill is taken). The temporal term $\bigcirc\left(x_{1}, p, . b ., 2\right)$ represents a loop with one cycle: $p$, an inter-repeat convex relation .b. (before) and a repetitions number 2 . (The first parameter, $x_{1}$, is an index.) Two repeats are associated to this loop, one before the other. The first repeat coincides 
with its cycle $(p)$; (this is formally defined later.) $\bigcirc\left(x_{2}, p, . b ., 2\right)$ is another loop with the same structure but different index than in the previous loop. Hence this loop also has two repeats, one before the other, where the first repeat is the cycle $p$. However the second repeat may be different from the second one in the previous loop.

Consider a sequence of 10 lunches, each consisting of a sandwich and two medication pills. Such situation is represented with a main loop $\bigcirc\left(x_{3},\left\langle s . b ., \bigcirc\left(x_{1}, p, . b ., 2\right)\right\rangle, . b ., 10\right)$. Its cycle is the related pair $\left\langle\mathrm{s}, . b ., \bigcirc\left(x_{1}, \mathrm{p}, . b ., 2\right)\right\rangle$, the inter-repeat relation is before and the repetitions number is 10 .

One loop is associated to many repeats, as many as the loop's repetitions number. Repeats are temporal terms, instances of the cycle. Hence they are in turn other structured temporal terms. Notice that repeats could have various types of similarities among them and still be representable by a single cycle, provided that the type of similarity is specified. In the present formalization we consider repeats to be similar if they have the same (temporal qualitative) shape. The concept of "same shape" is formally addressed in further sections.

The representation formalism used is pure first order logic with equality; sorts are identified with predicates. The logical connectives have the usual precedence rules; $\dot{V}$ is used for exclusive or. We describe the theory in an axiomatic way. The objects in this theory include temporal terms, (reified) temporal relations, numbers and (reified) propositions. Furthermore, there are four different types or sorts of temporal objects: convex intervals, related-pairs of temporal terms, related sets of temporal terms which we call rets, and loops. This is specified with the general axioms $g .1$ and $g .2$.

$$
\begin{gathered}
\text { g.1 } \forall x .(t t(x) \dot{\bigvee} \text { trelation }(x) \dot{\bigvee} \text { number }(x) \dot{\bigvee} \operatorname{prop}(x)) \\
\text { g.2 } \forall x .\left(t t ( x ) \supset \left(\begin{array}{l}
\text { interval }(x) \dot{\bigvee} \operatorname{relp}(x) \dot{\bigvee} \operatorname{ret}(x) \dot{\bigvee} \\
\operatorname{loop}(x)))
\end{array}\right.\right.
\end{gathered}
$$

We consider positive natural numbers, constants and variables. Number constants are directly written as such. The order relations $<$ and $=$ and the operations of addition and multiplication between numbers are assumed to be adequately axiomatized, with their usual meaning. We also use the cardinal transfinite number $\aleph_{0}$. Very briefly, $\aleph_{0}$ equals the cardinality of the natural numbers set. For example, it holds that $\forall n .\left(\right.$ number $\left.(n) \bigwedge\left(n \neq \aleph_{0}\right)\right) \supset n<\aleph_{0}$. Ordinal and cardinal transfinite arithmetic can be found for example in [8]. Number terms are used for two of the parameters in loops: the index and the repetitions number.

General temporal relations include Allen's convex interval relations [1]. Other relations are introduced in Section 2.2. The basic Allen's convex interval relations are written $. b ., . m ., . s ., d ., . o ., f$. and .eq. for before, meets, starts, during, overlaps, finishes and equals respectively. The inverse of a relation is also an "Allen relation" and is written for example.$b^{-1}$. for the inverse of before, (i.e. $a f$ ter). A set of relations is also an "Allen relation" and it represents the disjunction of those relations in the set. For example,.$i n$. = $\{. d ., . e q ., . s ., . f$.$\} . The predicate identifying Allen's convex interval$ relations is written as allen(_). Relations are reified (i.e. treated as objects); the relation $\mu$ between two temporal terms $t$ and $t^{\prime}$ is expressed as related $\left(t, t^{\prime}, \mu\right)$. The predicate related is assumed to be adequately axiomatized based on [3].

Atemporal assertions are reified propositions and appear in $H O L D S$ formulas. The formula $\operatorname{HOLDS}(t, \alpha)$ asserts that $\alpha$ is true during the interval/s that the temporal term $t$ represents. This is developed in Section 3.

\subsection{Temporal terms}

Recall that the different temporal terms are disjoint and consist of: (convex) intervals, related pairs, rets and loops. Essentially temporal terms represent structured temporal objects; i.e. they represent sets of intervals with a structure resulting from the relations among them.

Intervals are primitive objects. We include three kind of limit intervals: allime, since and until. Intuitively, alltime is one limit interval covering the whole time line; it has been defined elsewhere, for example [10]. A since interval starts at some finite point in the time line and extends forward; an until interval finishes at some point in the time line but does not have a finite beggining point.

A related-pair is the simplest structured temporal object. It essentially represents a (convex) binary relation or constraint between two temporal objects. But, in the axiomatization we treat this binary relation together with the related temporal objects as a related-pair object. The predicate identifying related-pairs terms is relp(_).

Three functors select related-pairs parameters. First and second provide respectively the first and second temporal object component in the pair, and rel provides the relation. We use an abbreviated notation borrowed from programming languages and express the first component of a related pair $p$ as $p .1$. Similarly $p .2$ and $p$.rel represent the second component and the relation component of $p$ respectively. This is expressed in Axiom rp.1:

\section{rp.1 related pair parameters}

$$
\forall p . \operatorname{relp}(p) \Leftrightarrow \exists t, t^{\prime}, \mu . t t(t) \bigwedge t t\left(t^{\prime}\right) \bigwedge \text { allen }(\mu) \bigwedge
$$$$
\left(p=\left\langle t, \mu, t^{\prime}\right\rangle \wedge t=p .1 \bigwedge t^{\prime}=p .2 \bigwedge \mu=\text { p.rel }\right) .
$$

The relation in the related-pair holds between the two temporal objects each considered "as a whole"; a temporal object is considered "as a whole" by considering its convexification via the $c v x$ functor. Briefly: a convexified temporal object is the smallest interval including all the subintervals in the object and is written $c v x\left(\_\right)$. Axiom $r p .2$ expresses the role of the relation in a related pair:

\section{rp.2 relation \\ $\forall p . \operatorname{relp}(p) \supset \operatorname{related}(\operatorname{cvx}(p .1), \operatorname{cvx}(p .2), p . r e l)$.}

Observation 1 A related pair is defined only if the two (convexified) components are related with the relation in the term. For example, for any temporal term $t,\langle t, . m ., t\rangle$ is not a related pair, since $\forall t . t t(t) \supset$ related $(\operatorname{cvx}(t), \operatorname{cvx}(t), . e q$.$) .$

Rets are sets of related pairs. Here we consider rets as non-empty finite lists of related pairs to simplify the presentation. We use the same notation for rets as lists in Prolog. Axiom r.l defines a ret and its parameters. Axioms $r .2$ and $r .3$ define the len and element functions, which provide the length and a particular element of the ret respectively.

\section{r.1 ret parameteres}

$$
\begin{aligned}
\forall r . \operatorname{ret}(r) \Leftrightarrow & ((\exists p . \operatorname{relp}(p) \bigwedge r=[p] \bigwedge p=\operatorname{first}(r)) \dot{\bigvee} \\
& (\exists p, s . \operatorname{relp}(p) \bigwedge \operatorname{ret}(s) \bigwedge r=[p \mid s] \bigwedge \\
& p=\operatorname{first}(r) \bigwedge s=\operatorname{rest}(r))) .
\end{aligned}
$$

r.2 len

a. $\forall p . \operatorname{relp}(p) \supset \operatorname{len}([p])=1$.

b. $\forall r, s, p .(\operatorname{ret}(r) \wedge r=[p \mid s]) \supset(\operatorname{len}(r)=1+\operatorname{len}(\operatorname{rest}(r))$.

r.3 element

$\forall r \cdot \operatorname{ret}(r) \supset(($ element $(r, 1)=f \operatorname{first}(r)) \wedge$

$((\forall n$. number $(n) \wedge n<\operatorname{len}(r)) \supset$

$(\operatorname{element}(r, n+1)=\operatorname{element}(\operatorname{rest}(r), n)))$. 
From these axioms it follows that a ret $r$ is composed of a finite number of related pairs.

Example 1 Let $i, j, k, l$ be intervals and $\mu, \nu$ Allen convex interval relations. Let $r=\left[\langle i, \mu, j\rangle \mid r^{\prime}\right]$, where $r^{\prime}=[\langle k, \nu, l\rangle]$, Then

$r=[\langle i, \mu, j\rangle,\langle k, \nu, l\rangle], \operatorname{len}\left(r^{\prime}\right)=1, \operatorname{len}(r)=\operatorname{len}\left(r^{\prime}\right)+1=2$,

element $(r, 1)=\operatorname{first}(r)=\langle i, \mu, j\rangle$, element $(r, 2)=$

element $\left(r^{\prime}, 1\right)=\langle k, \nu, l\rangle$

Temporal repetition: Loops This section presents the loops axioms, intermixed with examples, other axioms and clarifications. Recall that a loop is defined by four parameters: its index, its cycle, an inter-repeat relation and a repetitions number. Axiom $l .1$ defines a loop and its parameters.

\section{l.1 loop parameters}

$$
\begin{aligned}
& \forall l . l o o p(l) \Leftrightarrow \exists x, t, \mu, n .(\text { number }(x) \bigwedge t t(t) \bigwedge \text { allen }(\mu) \wedge \\
& n u m b e r(n) \bigwedge l=\bigcirc(x, t, \mu, n) \bigwedge x=\operatorname{index}(l) \bigwedge \\
& \left.t=\operatorname{cycle}(l) \bigwedge \mu=\operatorname{rel}(l) \bigwedge n=\operatorname{rep\_ num}(l)\right) .
\end{aligned}
$$

We propose that associated with a loop there are repeats (as many as the repetitions number), all of which have the same "shape" as the cycle's. "Same-shapeness" is used as a similarity criterion among repeats here. Other similarity relations could involve alternative qualitative considerations or quantitative information. For example, temporal terms could be required to have the same duration.

The predicate same_shape formalizes this concept, and is used in Axiom l.2. This is where our formalization connects with the intuition of a closed time structure in the sense that repeats are "the same". Indeed, we propose that a loop stands for many repeats, all with the same shape (and therefore representable by) the cycle. Additionally, the cycle itself (as a temporal term) represents a specific repeat, the first one. This is what Axiom $l .3$ expresses. Notice that the index is irrelevant with respect to the loop shape.

Same_shape is an equivalence relation; additionally the axioms sh. 1 to $s h .4$ hold.

$$
\begin{aligned}
& \text { sh.1 } \forall i, i^{\prime} .\left(\text { interval }(i) \bigwedge \text { interval }\left(i^{\prime}\right)\right) \Leftrightarrow \\
& \text { same_shape }\left(i, i^{\prime}\right) \text {. } \\
& \text { sh.2 } \forall p, p^{\prime} .\left(\operatorname{relp}(p) \wedge \operatorname{relp}\left(p^{\prime}\right)\right) \supset\left(\operatorname{same\_ shape}\left(p, p^{\prime}\right) \Leftrightarrow\right. \\
& \left(\text { same_shape }\left(p .1, p^{\prime} .1\right) \bigwedge \text { same_shape }\left(p .2, p^{\prime} .2\right) \wedge\right. \\
& \text { p.rel } \left.=p^{\prime} . r e l\right) \text { ). } \\
& \text { sh.3 } \forall r, r^{\prime} .\left(\operatorname{ret}(r) \bigwedge \operatorname{ret}\left(r^{\prime}\right)\right) \supset\left(\text { same_shape }\left(r, r^{\prime}\right) \Leftrightarrow\right. \\
& \left(\left(\operatorname{len}(r)=\operatorname{len}\left(r^{\prime}\right)\right) \bigwedge\right. \\
& \left(\text { same_shape }\left(\text { first }(r), \text { first }\left(r^{\prime}\right)\right)\right) \wedge \\
& \left.\left.\left((\text { len }(r)>1) \supset \text { same_shape }\left(\operatorname{rest}(r), \operatorname{rest}\left(r^{\prime}\right)\right)\right)\right)\right) \text {. } \\
& \text { sh.4 } \forall l, l^{\prime} \text {. }\left(\operatorname{loop}(l) \wedge \operatorname{loop}\left(l^{\prime}\right)\right) \supset\left(\text { same_shape }\left(l, l^{\prime}\right) \Leftrightarrow\right. \\
& \left(\text { same_shape }\left(\text { cycle }(l), \text { cycle }\left(l^{\prime}\right)\right) \wedge\right. \\
& \left.\left.\left(\operatorname{rel}(l)=\operatorname{rel}\left(l^{\prime}\right)\right) \wedge\left(\text { rep_num }(l)=\text { rep_num }\left(l^{\prime}\right)\right)\right)\right) \text {. }
\end{aligned}
$$

\section{Loops axioms - cont.}

\section{2 repeat existence}

$$
\begin{aligned}
& \forall l, n .\left(\text { loop }(l) \bigwedge \text { number }(n) \bigwedge\left(n \neq \aleph_{0}\right) \wedge\right. \\
& n \leq \text { rep_num }(l)) \supset(\exists r . t t(r) \bigwedge r=\operatorname{repeat}(l, n) \wedge \\
& \text { same_shape }(r, \text { cycle }(l))) .
\end{aligned}
$$

1.3 the cycle: first repeat

$$
\forall l \text {. } \operatorname{loop}(l) \supset(\text { cycle }(l)=\operatorname{repeat}(l, 1)) \text {. }
$$

Similarly to the case of related pairs, the relation parameter in the loop has a special role. Intuitively, the $n^{t h}$ repeat relates (as a whole) to the $(n+1)^{t h}$ repeat with the inter-repeats qualitative relation in the loop. This is formalized with Axiom l.4:

\section{4 inter-repeat relation}

$\forall l, n .\left(\operatorname{loop}(l) \wedge\right.$ number $\left.(n) \bigwedge n<\operatorname{rep\_ num}(l)\right) \supset$

related $(\operatorname{cvx}(\operatorname{repeat}(l, n)), \operatorname{cvx}(\operatorname{repeat}(l, n+1)), \operatorname{rel}(l))$.

\section{Example 2}

Let $i$ be an interval, $n$ be a finite number;

a. $\bigcirc(x, i, b ., n)$ represents a loop with the structure of $n$ repeats (intervals), one before the next. This corresponds to a non-convex interval with n subcomponents as defined in [11].

b. $\bigcirc\left(x, i, b ., \aleph_{0}\right)$ represents a loop with the structure of an interval before the next, occurring forever, with a first repetition denoted by the interval $i$. An example of such loop structure could be the sun rising every day, assuming that this will happen forever and that there was a first sunrise.

Let coffee and meeting be interval variables; $\bigcirc(x,\langle$ coffee. $o . \bigcirc$ (y,meeting.b., 3$)\rangle, . b ., 10)$ represents a loop with ten repetitions (each before the next one as a whole) where a coffee interval overlaps with three meetings (which occur one before the next).

To simplify the formalism, another loop axiom requires that no loop with repetitions number $\aleph_{0}$ can be within a loop cycle.

\subsection{A new temporal relation and composition of loops}

We present the definition of a new temporal relation between loops, primarily to illustrate the power of the formalism. We show how this relation compares with analogous relations defined in previous related work about "non-convex" intervals [11]. Further we present essentially the same concept but as a composition operation of two loops. This introduces a different perspective to the concept, leading to a more succinct way of expressing repetitive temporal patterns.

In previous sections we have dealt with relations between any temporal term when considering their convexification, i.e. considering terms as a whole. Many other relations exist between temporal terms when taking into account their subcomponents. Furthermore, it is possible to define relations between temporal terms which take into account their defining parameters, their inner structure, and so on.

Here we present one relation between two loops taking into account (all of) the respective repeats, and each repeat considered as a whole: " $A L L \mu$ ", where $\mu$ is an Allen convex interval relation. The $A L L \mu$ relation is one of the possible extensions of the relation "always R" between non-convex intervals as defined in [11], where " $\mathrm{R}$ " is an Allen relation. In the referred paper binary relations between non-convex intervals include those that take into account subintervals (such as "always R", "sometimes R"), and other relations which take the non-convex interval as a whole (via its convexification).

Briefly, we define the relation $A L L \mu$ to hold between two loops when the relation $\mu$ holds between their respective convexified repeats. Axioms are omitted for lack of space.

Example 3 Consider the situation "sometimes lunches meet seminars, sometimes lunches are before seminars". This type of example appears in [14] where a formalism based on Ladkin's non-convex intervals is introduced. One way of defining this situation in our language is with two loops, a lunches loop and a seminars loop, and relating them with the relation $A L L\left\{. m . \bigvee . b^{-1}.\right\}$.

This example is revisited below, under a different perspective, by using one single loop. 
Observation 2 If the cycles of two loops have some temporal term in common then for some Allen relation $\mu$, the $A L L \mu$ relation may be inconsistent.

Proof We prove this with an example, where the relation $A L L \mu$ cannot possibly hold. Let $l=\bigcirc(x, i, b, 3)$ and let $l^{\prime}=$ $\bigcirc\left(x^{\prime}, i, b^{-1}, 3\right)$. It is not possible that related $\left(l, l^{\prime}, A L L . b\right.$. $)$, since $i$ is the cycle of both loops (and therefore the first repeat for both loops), $l$ "evolves forward" (one repeat is before the next), $l$ ' "evolves backward", precluding that repeats of $l$ be before than repeats of $l^{\prime}$.

Composition of loops An alternative way of seeing relations such as $A L L \mu$ is by considering a new temporal loop which results from composing two other loops. We define a loop $l$ to be composed by loops $l_{1}$ and $l_{2}$ and the Allen relation $\mu$ when its $n^{t h}$ repeat is a related pair, where the first component in the pair is the $n^{\text {th }}$ repeat of loop $l_{1}$, the second component is the $n^{\text {th }}$ repeat of loop $l_{2}$ and the relation in the related pair is $\mu$. Formally,

Definition 1 composes $\left(l_{1}, l_{2}, \mu, l\right) \Leftrightarrow$ $\left(\operatorname{loop}\left(l_{1}\right) \wedge \operatorname{loop}\left(l_{2}\right) \wedge \operatorname{loop}(l) \bigwedge\right.$ allen $(\mu) \wedge$ rep_num $\left(l_{1}\right)=$ rep_num $\left(l_{2}\right)=\operatorname{rep\_ num}(l) \wedge$ $\forall n .\left(\right.$ number $(n) \bigwedge n \leq \operatorname{rep\_ num}(l) \wedge r_{1}=\operatorname{repeat}\left(l_{1}, n\right) \wedge$ $\left.\left.r_{2}=\operatorname{repeat}\left(l_{2}, n\right) \wedge r=\operatorname{repeat}(l, n)\right)\right) \supset r=\left\langle r_{1}, \mu, r_{2}\right\rangle$.

Theorem $1 \forall l_{1}, l_{2}, \mu .\left(\operatorname{loop}\left(l_{1}\right) \bigwedge \operatorname{loop}\left(l_{2}\right) \bigwedge\right.$ allen $\left.(\mu)\right) \supset(\exists l$. $\left(\operatorname{loop}(l) \wedge\right.$ composes $\left.\left.\left(l_{1}, l_{2}, \mu, l\right)\right) \Leftrightarrow \operatorname{related}\left(l_{1}, l_{2}, A L L \mu\right)\right)$.

This theorem illustrates the expressive power of loops in our language. Certain repetitive temporal patterns can be expressed succinctly with a single loop whose cycle is a structured temporal object. In contrast, if one considers interval cycle loops (analogous to nonconvex intervals), several of such loops are required, together with the $A L L \mu$ relation. Under this perspective, we review Example 3;

Example 4 "Sometimes lunches meet seminars, sometimes lunches are before seminars" can be expressed with the loop $\bigcirc(x$, 〈seminar $\left\{. m . \bigvee \cdot b^{-1}.\right\}$ lunch $\left.\rangle, b ., n\right)$. This loop is the composition of the two loops lunches and seminars, and relation $\left\{. m . \bigvee \cdot b^{-1}.\right\}$

Clearly, Observation 2 above about the $A L L \mu$ relation can be seen as referring to the (non)-existence of such composed loop, given the equivalence in Theorem 1. Other interesting results follow from considering composed loops and the interconnection between the loops inter-repeat relation and the composing relation.

\section{Formulas: atemporal assertions associated to temporal terms}

We next consider how one can reason with atemporal assertions that are true during temporal terms. In common with previous work $[2,17]$ we introduce $H O L D S$ formulas, where an atomic formula consists of the HOLDS predicate with two arguments: a temporal term as defined in previous sections and a reified proposition. Intuitively $\operatorname{HOLDS}(t, \alpha)$, means that $\alpha$ is true during $t$.

We treat atemporal assertions as Allen's properties in that something is true during an interval if it is true during all the intervals within. This extends nicely to the behaviour of atemporal assertions during composite temporal terms, since we require that an assertion is true during a temporal term if it is true during all its subcomponents. See Axiom H.1 and Theorem 2.
Composed formulas are related with the usual logical connectives $\{\neg, \bigvee, \wedge \supset\}$. Similarly to the referred work we apply the operators not, and, or and implies to atemporal assertions and we write for example $\operatorname{HOLDS}(t, \operatorname{and}(\alpha, \beta))$ as a shorthand for $\operatorname{HOLDS}(t, \alpha) \wedge \operatorname{HOLDS}(t, \beta))$. The interpretation we give to negation is that of strong negation, akin to the negation for properties in Allen's formalization. Hence $\operatorname{not}(\alpha)$ holds during a temporal term if $\alpha$ does not hold during any of the temporal term subcomponents. The "strong negation" is not equivalent but it implies the "weak negation"; with weak negation $\operatorname{not}(\alpha)$ would hold during a temporal term if $\alpha$ does not hold during the temporal term itself. See Axiom H.2 and Theorem 3. We use the predicate subcomponent to shorten the axioms presentation. Intuitively: intervals which are in. an interval make up its subcomponents; the two components in a related pair are subcomponents of the related pair; the first and rest of a ret are its subcomponents and the repeats associated to a loop are its subcomponents. Furthermore, subcomponents of subcomponents are also subcomponents of a temporal term.

\section{H.1 proposition variable}

$$
\begin{aligned}
& \forall t, x .(t t(t) \bigwedge \text { prop_var }(x)) \supset \\
& (H O L D S(t, x) \Leftrightarrow \\
& \left(\forall t^{\prime} .\left(t t\left(t^{\prime}\right) \bigwedge \text { subcomponent }\left(t^{\prime}, t\right)\right) \supset\right. \\
& \left.\left(\exists t^{\prime \prime} . \text { subcomponent }\left(t^{\prime \prime}, t^{\prime}\right) \bigwedge H O L D S\left(t^{\prime \prime}, x\right)\right)\right) .
\end{aligned}
$$

H.2 not

$$
\begin{aligned}
& \forall t, \alpha .(t t(t) \bigwedge \text { prop }(\alpha)) \supset(\operatorname{HOLDS}(t, \operatorname{not}(\alpha)) \Leftrightarrow \\
& \left(\forall t^{\prime} . t t\left(t^{\prime}\right) \bigwedge \text { subcomponent }\left(t^{\prime}, t\right) \supset\right. \\
& \left.\left.\neg \operatorname{HOLDS}\left(t^{\prime}, \alpha\right)\right)\right) .
\end{aligned}
$$

H.3 and

$$
\begin{aligned}
& \forall t, \alpha, \beta .(t t(t) \bigwedge \operatorname{prop}(\alpha) \wedge \operatorname{prop}(\beta)) \supset \\
& (\operatorname{HOLDS}(t, \operatorname{and}(\alpha, \beta)) \Leftrightarrow \\
& (\operatorname{HOLDS}(t, \alpha) \bigwedge \operatorname{HOLDS}(t, \beta))) .
\end{aligned}
$$

Axioms for the proposition expressions with functors or and implies are analogous to Axiom H.3. Theorem 2 follows from Axiom H.1, see [2], page 130; it follows as well that it applies to any proposition expression (and not only to proposition variables). Theorem 3 shows a result akin to how negation behaves for properties in Allen's formalizartion. An analogous result holds for related pairs, rets and loops. Theorem 4 illustrates how binary logical operators "move freely within" a HOLDS formula. (Proofs are not included for lack of space.)

Theorem $2 \forall t, \alpha \cdot(t t(t) \wedge p r o p(\alpha)) \supset$ $\left(\operatorname{HOLDS}(t, \alpha) \Leftrightarrow\left(\forall t^{\prime} . t t\left(t^{\prime}\right) \bigwedge\right.\right.$ subcomponent $\left(t^{\prime}, t\right) \supset$ $\left.\left.\operatorname{HOLDS}\left(t^{\prime}, \alpha\right)\right)\right)$.

Theorem $3 \forall i, \alpha$. (interval $(i) \wedge p r o p(\alpha)) \supset$ $(\operatorname{HOLDS}(i, \operatorname{not}(\alpha)) \supset \neg \operatorname{HOLDS}(i, \alpha))$.

Theorem $4 \forall t, \alpha, \beta .(t t(t) \bigwedge p r o p(\alpha) \bigwedge p r o p(\beta)) \supset$ $((\operatorname{HOLDS}(t, \alpha) \wedge \operatorname{HOLDS}(t$, implies $(\alpha, \beta))) \supset$ $\operatorname{HOLDS}(t, \beta))$.

Two temporal terms were defined to have the "same shape" with the predicate same_shape, and such concept essentially implies a renaming of variables. This concept can be extended, even to hold between temporal terms of different types. Intuitively, "equivalentlyshaped" temporal terms include same-shaped ones, and additionally, any two temporal terms which have the same structure at the interval level. The following theorems refer to some of these situations. 
Theorem $5 \forall x, i, \mu, \alpha . \quad($ interval $(i) \bigwedge$ allen $(\mu) \bigwedge p r o p(\alpha)) \supset$ $(\operatorname{HOLDS}(\bigcirc(x, i, \mu, 2), \alpha) \Leftrightarrow \exists j$. interval $(j) \bigwedge$ $\operatorname{HOLDS}(\langle i, \mu, j\rangle, \alpha) \Leftrightarrow \exists j$. interval $(j) \wedge \operatorname{HOLDS}([\langle i, \mu, j\rangle], \alpha))$.

The next result we show applies to forward and backward loops, where a loop $l$ is forward iff its inter-repeat relation $\mathrm{rel}(l)$ is a subset of $\{. b ., . m ., . e q$.$\} , and a loop is backward iff the inter-repeat relation$ is a subset of $\left\{. b .^{-1}, . m .^{-1}, . e q.\right\}$. The subset relation among Allen relations is defined as follows.

Definition $2 \forall \mu, \mu^{\prime}$. (allen $(\mu) \bigwedge$ allen $\left.\left(\mu^{\prime}\right)\right) \supset$ $\operatorname{subset}\left(\mu, \mu^{\prime}\right) \Leftrightarrow\left(\forall i, i^{\prime}\right.$. interval $(i) \bigwedge$ interval $\left(i^{\prime}\right) \supset$ $\left.\left(\operatorname{related}\left(i, i^{\prime}, \mu\right) \supset \operatorname{related}\left(i, i^{\prime}, \mu^{\prime}\right)\right)\right)$.

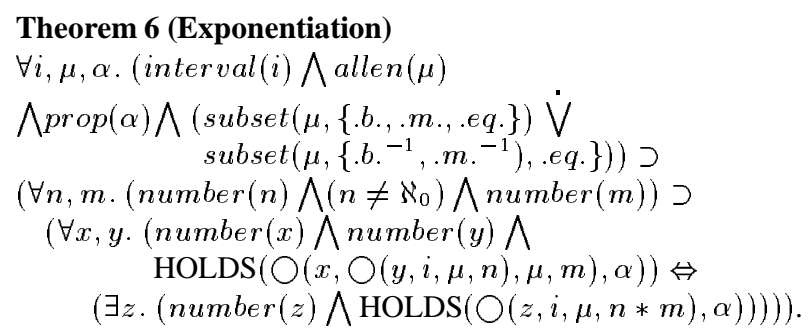

This result is akin to the exponentiation theorem which can be expressed in regular languages as $\left(a^{*}\right)^{*}=a^{*}$, or if they are finite strings as $\left(a^{n}\right)^{m}=a^{(n * m)}$. Notice that the infinite case is covered in the theorem by having (only the outer) loop with repetitions number $\aleph_{0}$, i.e. a result akin to $\left(a^{n}\right)^{*}=a^{*}$. The exponentiation property does not apply to loops which do not evolve forward or backward such as for example having overlaps as the inter-repeat relation.

\section{Discussion and Related Work}

We have presented an approach to formally characterize time with structured temporal objects. Based on convex intervals, we have defined temporal objects which are composed themselves of temporal terms related in certain ways. This is analogous to how complex objects are composed of different parts and sub-parts. In particular we deal with temporal repetition, represented with time loops. A time loop captures in one cycle the core of what is repeated; a loop cycle consists of an interval or a structured object (including other subloops). The loop's inter-repeat relation specifies the relation that holds between each repetition; it can be any interval relation. Hence, overlapping intervals or sequences of intervals going "backwards" in time are possible. We have also formalized loops where the repetitions number of the loop is the transfinite number $\aleph_{0}$. The approach is formalized in first order logic. The language includes temporal terms and a HOLDS predicate which has two arguments: a structured temporal term and a (reified) proposition which holds during the temporal term. Several results are presented which intuitively show that the theory behaves correctly. Our language allows for the compact representation of repeated temporal entities. This would be appropriate for situations where similarities between repeats are known, which is the case in many practical applications. Hence we provide an abstraction mechanism for temporal objects, and so propose (and allow) a further degree of structure in temporal knowledge bases. We are aware of one medical system [5] that has recently incorporated these ideas based on our previous version of the language [7]. Examples such as lunches meet seminars or lunches are before seminars are covered within one loop: $\bigcirc\left(x,\left\langle\right.\right.$ seminar $\left\{. m . \bigvee \cdot b^{-1}.\right\}$ lunch $\left.\rangle, . b ., n\right)$. This type of example appears in [14]. o express the above example with non-convex intervals instead of loops, there would exist two different non-convex intervals, "seminars" and "lunches", one corresponding to the set of seminars and the other to the lunches. This example would then be expressed as a relation between correlated pairs using non-convex intervals: each single seminar meets or is after each single "closest" lunch. In the presented formalism the linking relation between "closest" seminars and lunches is made explicit, as being part of a cycle given in the loop construct.

As mentioned above, work in formalizing calendars and time units also deals with structured time. However, such work is focused on calendar-based notions, and does not attempt to present a formalization of what (structured) temporal repetition essentially is. We here propose a generic paradigm to treat time; we formalize it with temporal objects which include structure (via qualitative relations in this document). Furthermore we propose a succinct way of representing repetitive temporal patterns.

Current and future research includes exploring results that follow from the axiomatization, formally proving the consistency of the theory and a study of the suitability of this formalism to reason within practical applications. Constraint propagation algorithms which apply to the defined temporal objects and profit from the specific (one cycle based) structure that we propose are also part of future research. An extension is to incorporate quantitative information into the formalism. This would allow one for example to quantitatively specify calendars and granularity systems in general.

\section{REFERENCES}

[1] J. F. Allen, 'Maintaining knowledge about temporal intervals', Соттиnications of the ACM, 26(11), 832-843,(1983).

[2] J. F. Allen, 'Towards a general theory of action and time', Artificial Intelligence, 23, 123-154,(1984).

[3] J. F. Allen and P. J. Hayes, 'A common-sense theory of time', in Proc. of the Ninth IJCAI-85, pp. 528-531, Los Angeles, CA, (1985).

[4] C. Bettini, X. S. Wang, and S Jajodia, 'A general framework for time granularity and its application to temporal reasoning', Annals of Mathematics and Artificial Intelligence, 22(1,2), 29-58, (1998).

[5] S. Chakravarty and Y. Shahar, 'A constraint-based specification of periodic patterns in time-oriented data', in TIME'99, pp. 29-40, FL, USA, (1999). IEEE Computer Society.

[6] D. Cukierman and J. Delgrande, 'Expressing time intervals and repetition within a formalization of calendars', Computational Intelligence, 14(4), 563-597, (November 1998).

[7] D. Cukierman and J. Delgrande, 'Towards a formal characterization of temporal repetition with closed time', in TIME'98, pp. 140-147, FL, USA, (May 1998). IEEE Computer Society Press.

[8] K. Hrbacek and T. Jech, Introduction to Set Theory, Second Edition, Revised and expanded, Marcel Dekker, Inc., New York, 1984.

[9] F. Kabanza, J-M Stevenne, and P. Wolper, 'Handling infinite temporal data', in PODS'90, pp. 392-403, Tenessee, (1990).

[10] P. B. Ladkin, 'Primitives and units for time specification', in AAAI-86, pp. 354-359, (1986).

[11] P. B. Ladkin, 'Time representation: a taxonomy of interval relations', in AAAI-86, pp. 360-366, (1986).

[12] B. Leban, D. D. McDonald, and D. R. Forster, 'A representation for collections of temporal intervals', in AAAI-86, pp. 367-371, (1986).

[13] G. Ligozat, 'On generalized interval calculi', in AAAI-91, pp. 234-240, (1991).

[14] R. A. Morris, W. D. Shoaff, and L. Khatib, 'Path consistency in a network of non-convex intervals', in IJCAI-93, pp. 655-660, (1993).

[15] W. H. Newton-Smith, The Structure of Time, Routledge and Kegan Paul Ltd. publishers, 1980.

[16] M. Niézette and J. Stévenne, 'An efficient symbolic representation of periodic time', in Proc. of the 1st. International Conference on Information and Knowledge Management, Baltimore, Maryland, (1992).

[17] Y. Shoham, Reasoning About Change: Time and Causation from the Standpoint of Artificial Intelligence, The MIT Press, Mass., 1988.

[18] A. Tuzhilin and J. Clifford, 'On periodicity in temporal databases', Information Systems, 20(8), 619-639, (1995). 